\title{
True blue: a puzzling case of persistent cyanosis in a young child
}

\author{
Michelle Porepa MD, Lee Benson MD, David E. Manson MD, Jeremy N. Friedman MBChB
}

A 2-year-old twin boy was referred to a cardiology clinic for long-standing cyanosis. He had been born to a healthy 36-year-old mother. The diamnionic, dichorionic twin pregnancy had been complicated by maternal cholestasis, which had been treated with ursodiol. Amniocentesis had been performed, and the findings were normal.

The twins had been delivered by cesarean section at $34+6$ weeks' gestation. Our patient's Apgar scores were 8 at 5 minutes and 9 at 10 minutes. His birth weight was $2225 \mathrm{~g}$. He required tracheal intubation shortly after birth because of low oxygen saturation levels. An initial radiograph of his chest showed an increased cardiothoracic ratio, which raised suspicion of congenital heart disease. An echocardiogram appeared normal except for a restrictive patent arterial duct. The tracheal tube was removed shortly thereafter.

He remained on low-flow supplemental oxygen for 4 weeks. His oxygen saturation levels were in the low $90 \%$ range, falling to the mid- $80 \%$ range without oxygen. The differential diagnosis for his dependence on supplemental oxygen included patent ductus arteriosus, neonatal chronic lung disease and mild pulmonary hypertension (although this last condition was not confirmed on an echocardiogram). The boy was given nebulized budesonide therapy and weaned off oxygen. He was discharged home with no oxygen therapy. His twin sibling had an unremarkable postpartum course and had no need for oxygen therapy.

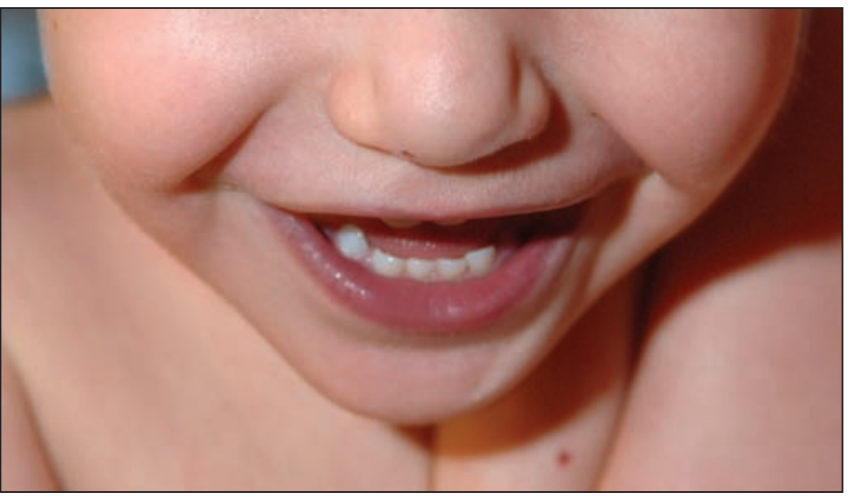

Figure 1: Photograph showing clinically evident cyanosis in the lips of a 2-year-old boy with hypoxemia (oxygen saturation level in the low $70 \%$ range).

\section{Key points}

- Persistent cyanosis, even in an otherwise well child, should be investigated, since there are important cardiac, respiratory, vascular and hematologic causes.

- Pulmonary arteriovenous fistula should be suspected when the workup of persistent cyanosis indicates the presence of a noncardiac right-to-left shunt (e.g., failed hyperoxia test, positive bubble echocardiogram).

- Early diagnosis and surgical correction are important to avoid thromboembolic complications.

Over time, the patient showed normal growth, development and energy levels. His growth consistently measured in the 5th percentile for weight and the 10th percentile for height, and matched that of his twin sister.

During his second year, his parents observed a persistently blue tinge around his lips and nail beds. He showed no associated shortness of breath, loss of consciousness or distress. A general pediatrician who was following him at the time ruled out methemoglobinemia and arranged a follow-up radiograph of the boy's chest. The radiograph was reported as normal. The boy's oxygen saturation levels had not been documented since his discharge from the neonatal intensive care unit.

On examination in the cardiology clinic, the boy looked well and was in no distress. His heart rate was 114 beats/min and his blood pressure $90 / 43 \mathrm{~mm} \mathrm{Hg}$ in the left arm. His oxygen saturation level was $73 \%$ in room air. His height was in the fifth percentile at $81 \mathrm{~cm}$, and he weighed $10 \mathrm{~kg}$, also in the fifth percentile. He had visible cyanosis of his mucous membranes and lips (Figure 1) and clubbing of his fingers and toes (Figure 2). He had a very short grade $1 / 6$ systolic ejection murmur that was best heard at the upper left sternal border. The rest of the findings on examination were normal.

A radiograph of the chest showed increased opacity in the left hilum (Figure 3). An electrocardiogram showed normal sinus rhythm and no evidence of chamber enlargement or ischemic changes. In a hyperoxia test using $100 \%$ oxygen and

From the Department of Pediatrics, Faculty of Medicine (Porepa), University of Toronto; and the Divisions of Cardiology (Benson) and Pediatric Medicine (Friedman), Department of Pediatrics, and the Department of Diagnostic Imaging (Manson), Hospital for Sick Children, Toronto, Ont. 


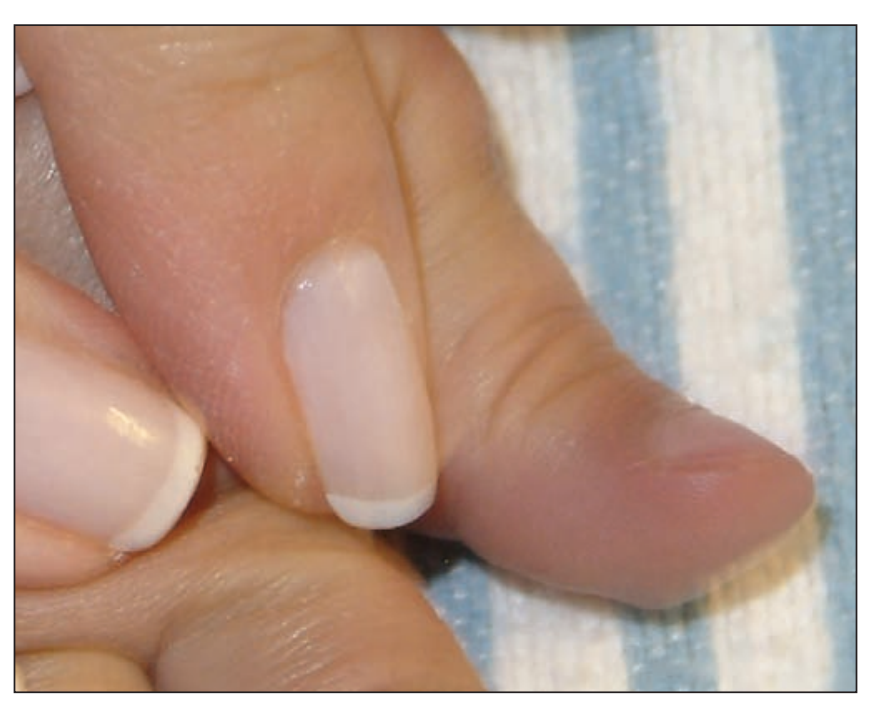

Figure 2: Photograph showing the patient's clubbed fingers next to the examiner's normal fingers.

an oxygen hood, the patient's oxygen saturation level, measured by pulse oximetry, remained at $73 \%$. His hemoglobin level was 168 (normal 110-140) g/L, his hematocrit level was 0.52 (normal $0.35-0.42$ ), and his arterial oxygen tension was $39 \mathrm{~mm} \mathrm{Hg}$. A second echocardiogram showed normal intracardiac anatomy. The working diagnosis was an extracardiac right-to-left shunt.

An agitated saline bubble contrast solution was injected through the patient's peripheral vein during an echocardiogram (a procedure known as a "bubble echocardiogram"). The bubbles were seen in his left atrium in the absence of a patent oval foramen. A computed tomography scan of the chest showed normal lung parenchyma but a large fistula from the left pulmonary artery to the left atrium. The patient was taken to the cardiac catheterization laboratory for embolization of the fistula with an occluding device (Figure 4). After the procedure, there was minimal flow in the fistula, and the boy's oxygen saturation level increased to $100 \%$ in room air.

Although the boy's rate of growth remained the same over the 6 months after the procedure, his parents reported a markedly improved energy level.

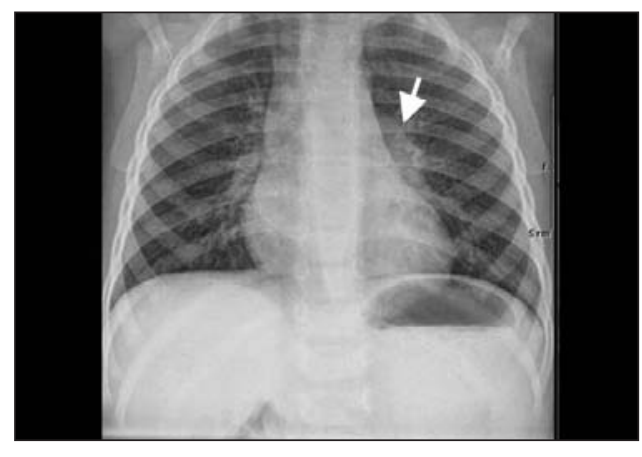

Figure 3: Chest radiograph showing increased opacity in the left hilum (white arrowhead).

\section{Discussion}

The fistula between our patient's pulmonary artery and the left atrium was a rare isolated congenital malformation. However, cyanosis is a common presentation in a variety of potentially life-threatening diseases in pediatrics. A good basic approach to the differential diagnosis of cyanosis in a child is therefore necessary.

Cyanosis can present at any age in childhood and may be intermittent or persistent. Central cyanosis typically involves the lips, tongue, mucous membranes and nail beds, whereas peripheral cyanosis involves the fingers and toes. Pseudocyanosis is a bluish tinge to the skin or mucous membranes that is not associated with hypoxemia or peripheral vasoconstriction.

The ability to recognize cyanosis depends on several factors, including skin tone, room lighting, the level of deoxygenated hemoglobin, the total hemoglobin concentration and the general state of perfusion. Checking pulse oximetry will distinguish cyanotic from pseudocyanotic states, although the accuracy of oximetry is influenced by alterations in $\mathrm{pH}$ levels, hemoglobin levels, arterial carbon dioxide tension, peripheral perfusion and movement artifact. Measuring the oxygen tension from arterial blood (taken from the right radial artery in a neonate) is the most valid reflection of arterial oxygenation status. ${ }^{1}$

In our discussion we focus on persistent, central cyanosis, which was the hallmark of our case presentation. Cardiac, respiratory, vascular and hematologic diseases can all produce persistent cyanosis (Table 1). An assessment for associated clinical features will help clarify the cause. Findings that suggest respiratory disease include respiratory distress, apnea and clubbing. The presence of a significant murmur, cardiomegaly and hepatomegaly point toward a cardiac cause. Simple diagnostic tests such as a radiograph of the chest, an electrocardiogram, an arterial blood gas test and a hyperoxia test are generally helpful. ${ }^{1}$ Our patient's presentation of cyanosis and clubbing without respiratory distress limited the likely diagnostic possibilities to a vascular abnormality or an atypical presentation of a congenital heart lesion.

A fistula from the pulmonary artery to the left atrium is an unusual form of cyanotic congenital heart disease that can also be thought of as an atypical variant of a pulmonary arteriovenous fistula. ${ }^{2}$ Autopsy reports from Johns Hopkins Hospital showed only 3 cases of pulmonary arteriovenous malfor-

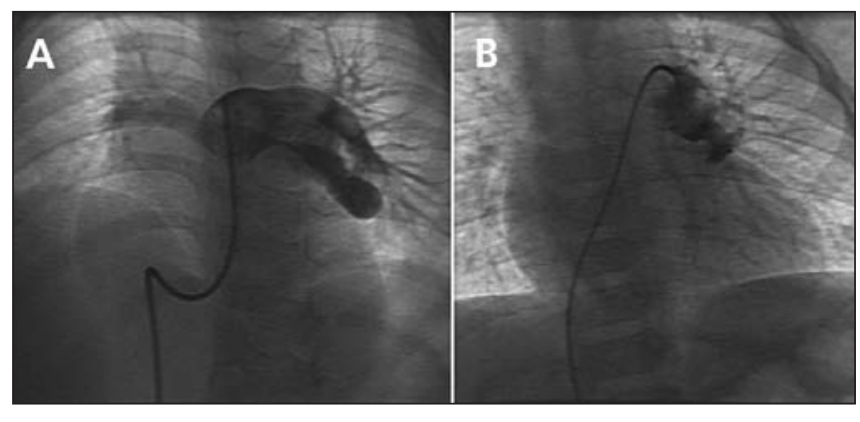

Figure 4: Angiographic images showing $(A)$ the fistula between the left pulmonary artery and the left atrium, before embolization, and (B) the fistula embolized with an occluding device. 
mations in 15000 autopsies. $^{3}$ The Mayo Clinic reported seeing 63 cases over 20 years ending in 1972, and 38 cases over 8.5 years ending in 1981, reflecting annual incidence figures of 3.2 and 4.5 cases. $^{3}$ Ten percent of cases are identified in infants or children. The incidence gradually increases among patients in their 50s and 60s. Pulmonary arteriovenous fistula can be an isolated congenital malformation but is more commonly (in $70 \%$ of cases) associated with hereditary hemor- rhagic telangiectasia - an autosomal-dominant syndrome characterized by multiple telangiectasias, recurring hemorrhages and vascular dysplasia. ${ }^{3}$ Acquired forms are rare and may be secondary to juvenile hepatic cirrhosis, pulmonary schistosomiasis, metastatic thyroid carcinoma or a postoperative complication. ${ }^{4}$

Clinicians should suspect pulmonary arteriovenous fistula in children with cyanosis in whom initial tests do not reveal

Table 1: Differential diagnosis of persistent central cyanosis in children

\begin{tabular}{|c|c|c|}
\hline Category of disorder & Diagnosis & Notes \\
\hline Vascular abnormality & $\begin{array}{l}\text { Extracardiac right-to-left shunt } \\
\text { - Pulmonary artery to left atrium } \\
\text { - Left superior vena cava to left atrium } \\
\text { - Inferior vena cava to left atrium } \\
\text { - Pulmonary arteriovenous malformations }\end{array}$ & $\begin{array}{l}\text { - Deoxygenated blood travels directly to the left } \\
\text { atrium, left ventricle or aorta instead of through } \\
\text { the lungs } \\
\text { - Pulmonary arteriovenous malformations are } \\
\text { commonly associated with hereditary } \\
\text { hemorrhagic telangiectasia } \\
\text { - Assess family history, bleeding history and } \\
\text { presence of telangiectasias }\end{array}$ \\
\hline
\end{tabular}

*Hyperoxia test: arterial oxygen tension is measured in blood taken from the right radial artery (preductal) while the patient breathes $100 \%$ oxygen for 10 minutes. The preductal oxygen tension rarely exceeds $150 \mathrm{~mm} \mathrm{Hg}$ in the presence of a right-to-left shunt (cyanotic heart disease) and usually exceeds this value in pulmonary disease. (A surrogate measure involves the use of a transcutaneous oxygen monitor to assess a rise in response to increased inspired oxygen concentration.) 
cardiac abnormalities. ${ }^{4}$ A review of cases shows a range of clinical presentations, including severe forms in newborns and a milder, less lethal form in older patients. Numerous case reports have documented fistulas from the pulmonary artery to the left atrium in patients who present for the first time in their teens with late-onset cyanosis and few other symptoms. ${ }^{5}$ Cyanosis of varying degrees, clubbing, shortness of breath, heart failure and polycythemia are cardinal symptoms and signs. ${ }^{4}$

Currently the best noninvasive diagnostic test is contrast bubble echocardiography, in which agitated saline solution is injected into a peripheral vein. The capillary alveolar filter should not allow the passage of these microbubbles through the lungs. Detection of bubbles in the left atrium therefore suggests a right-to-left shunt that has bypassed the lungs. Early appearance of bubbles in the left atrium (within 3 cardiac cycles of their appearance in the right atrium) suggests the presence of a patent oval foramen. Late-appearing bubbles, as in our patient's case, suggest extracardiac right-to-left shunting. Newer imaging modalities, such as multidetector-row computed tomography and various cardiac magnetic resonance imaging techniques, can provide accurate anatomic information about extracardiac vascular abnormalities and provide an alternative to more invasive angiography. The clinical utility and benefits of such modalities continue to be defined. ${ }^{6}$

It is important that pulmonary arteriovenous fistulas be suspected clinically when evaluating persistent, central cyanosis, since definitive diagnosis and therapy are readily available. The aim of therapy is early occlusion of the fistula to prevent serious thromboembolic complications such as stroke or cerebral abscess secondary to paradoxical embolization. ${ }^{2}$ Treatment options depend on the location, size and number of lesions. The interventional approach of catheter occlusion using various tools has proven to be successful ${ }^{2}$ and was the treatment chosen for our patient.

This article has been peer reviewed.

Competing interests: None declared.

\section{REFERENCES}

1. Tingelstad J. Non-respiratory cyanosis. Pediatr Rev 1999;20:350-2.

2. Jimenez M, Fournier A, Choussat A. Pulmonary artery to the left atrium fistula as an unusual cause of cyanosis in the newborn. Pediatr Cardiol 1989;10:216-20.

3. Gossage JR, Kanj G. Pulmonary arteriovenous malformations: a state of the art review. Am J Respir Crit Care Med 1998;158:643-61.

4. Gianisella RB, Rossi Filho RI, Zielinsky P. Diagnosis and therapeutics of pulmonary arteriovenous fistula in childhood. Case report and review of the literature. Arq Bras Cardiol 2001;77:274-81.

5. Krishnakumar N, Krisha MR, Tharakan JA. Right pulmonary artery to left atrial fistula: a differential diagnosis of cyanosis without structural heart disease. J Invasive Cardiol 2007;19:401-2.

6. Oguz B, Haliloglu M, Karcaaltincaba M. Paediatric multidetector CT angiography: spectrum of congenital thoracic vascular anomalies. Br J Radiol 2007;80:376-83.

The section Cases presents brief case reports that convey clear, practical lessons. Preference is given to common presentations of important rare conditions, and important unusual presentations of common problems. Articles start with a brief summary (100 words) outlining the case and its relevance to a general audience. The case presentation follows (500 words maximum) as well as a discussion of the underlying condition (1000 words maximum). Generally, up to 5 references are permitted and visual elements (e.g., tables of the differential diagnosis, clinical features or diagnostic approach) are encouraged. Written consent from patients for publication of their story is a necessity and should accompany submissions. See information for authors at www.cmaj.ca.

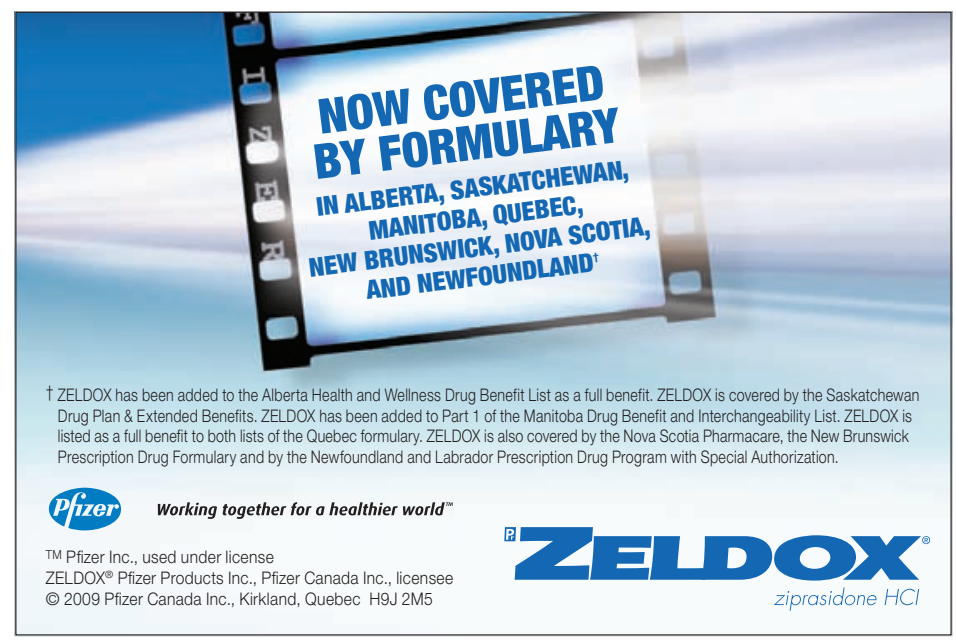

